\title{
Prescribing pattern of nutraceuticals in pediatric outpatient department at tertiary care teaching hospital
}

\author{
Madhuri Kulkarni, Priyanka Ambekar*, Siddharth Athawale
}

Department of Pharmacology, Government Medical College, Aurangabad, Maharashtra, India

\author{
Received: 04 February 2020 \\ Revised: 29 April 2020 \\ Accepted: 30 April 2020 \\ *Correspondence: \\ Dr. Priyanka Ambekar, \\ Email: priyankaambekar5@gmail.com
}

Copyright: () the author(s), publisher and licensee Medip Academy. This is an open-access article distributed under the terms of the Creative Commons Attribution Non-Commercial License, which permits unrestricted non-commercial use, distribution, and reproduction in any medium, provided the original work is properly cited.

\begin{abstract}
Background: Nutraceuticals use is increasing in a health-conscious society. Because of varying lifestyle diseases, people have changed their outlook towards nutraceuticals. The objective of the study was to study the prescription pattern of nutraceuticals by doctors in paediatric outpatient department of government medical college and hospital, Aurangabad, Maharashtra, India.

Methods: This study was done between November 2018 to March 2019. Total of 156 patients were included in the study after obtaining written informed consent from parents of patients. The data was gathered, analysed and results were concluded.

Results: Total 156 patients were prescribed nutraceuticals in paediatric outpatient department of which $51.92 \%$ female patients and $48.78 \%$ male patients received nutraceuticals. In this study, $62 \%$ patients received vitamins and $38 \%$ patients received vitamins and minerals. $67.31 \%$ patient receiving nutraceuticals were above the age of 6 years and $32.69 \%$ patients were under the age of 6 years. Among patients receiving nutraceuticals, B complex was received $55.77 \%$. After that calcium lactate + vitamin D tablet were prescribed $21.80 \%$. Ferrous sulfate + folic acid was received by $8.33 \%$ patients. Calcium carbonate + vitamin D3 were received by $7.69 \%$ patients. Folic acid tablet was received by $5.13 \%$ patients and poly-vitamin tablet by $1.28 \%$ patients. $100 \%$ prescriptions were prescribed in generic names.

Conclusions: Nutraceuticals are being increasingly perceived as beneficial to health and are being continuously used in the treatment. The future of nutraceuticals is bright and can transform healthcare in a developing country like India.
\end{abstract}

Keywords: Dietary supplements, Drug utilization study, Nutraceuticals

\section{INTRODUCTION}

The consciousness towards healthy diet has raised in population, because knowledge of effect of diet on human health has increased. People all over the world are frustrated with the expensive, hi-tech, disease treatment approach prominent in modern medicine, so the consumer is seeking alternative beneficial products and care like nutraceuticals. ${ }^{1}$ The term "nutraceutical" was originally coined by Dr. Stephen L. DeFelice, founder and chairman of the foundation of innovation medicine
(FIM), Crawford, New Jersey from "nutrition" and "pharmaceutical" in 1989. Health Canada modified its meaning and defined it as "a product isolated or purified from foods, and generally sold in medicinal forms not usually associated with food and demonstrated to have a physiological benefit or provide protection against chronic disease." 2 Nutraceutical is a marketing term developed for nutritional supplement that is sold with the intent to treat or prevent disease and thus has no regulatory definition. nutraceutical is any substance that may be considered a food or part of a food and provides 
medical or health benefits, encompassing, prevention and treatment of diseases. Presently over 470 nutraceutical and functional food products are available with documented health benefits. Nutraceuticals and functional foods have received considerable interest because of their presumed safety and potential nutritional and therapeutic effects. The global nutraceutical market size is estimated between 30 and 60 billion US\$, with Japan, US, and Europe occupying the biggest share. ${ }^{3}$ There is increasing market and use of nutraceuticals in United States, with estimated sales of over 21 billion dollars for the year $2006 .^{4}$

A nutraceutical is isolated from food stuff and it can be sold in the form of powders; pills and other forms. ${ }^{5}$ Nutraceuticals are helpful to complement nutrition in healthy subjects as well as patients. ${ }^{6}$ These products may range from isolated nutrients, dietary supplements and specific diets to genetically engineered designer foods and herbal products. $^{7}$ The Indians, Chinese and Sumerians are just a few civilizations that have provided evidence suggesting the food can be effectively used as a medicine. Indian nutraceutical market was estimated to be USD 1 billion in 2008. Global nutraceutical market is of USD 117 billion, of which India had less than $1 \%$ share in 2011.8

With the passage of the dietary supplement health and education act of 1994, the definition of nutraceuticals has been expanded to include vitamins, minerals, herbs and other botanicals, amino acids and any dietary substance for use by humans to supplement the diet by increasing total dietary intake and subsequently increased the use of nutraceuticals dramatically. ${ }^{9}$

There is a slight difference between the functional foods and nutraceuticals. When food is being cooked or prepared using "scientific intelligence" with or without knowledge of how or why it is being used, the food is called "functional food". Thus, functional food provides the body with the required number of vitamins, fats, proteins, carbohydrates, etc. needed for its healthy survival. When functional food aids in the prevention and/or treatment of disease(s) and/or disorder(s) other than anaemia, it is called a nutraceutical. ${ }^{10,11}$

Nutraceuticals are the emerging class of natural products that makes the line between food and drugs to fade (Adelaja and Schilling 1999). Although the use of nutraceuticals by people has a long history, only recently scientifically supported nutritional and medical evidence has allowed nutraceuticals to emerge as being potentially effective (Dillard and German 2000). ${ }^{12}$

Differences in demographic and behavioural characteristics between nutraceuticals users and nonusers would demonstrate the importance of including supplement assessment in both the planning and analysis of any epidemiologic study of diet or lifestyle characteristics and health. ${ }^{13}$
Nutraceutical is regarded as the bio active substance and the constituents are either of known therapeutic activity or are chemically defined substance generally accepted to contribute substantially to the therapeutic activity of the drug. ${ }^{14}$

There is little available data in India on prescribing trends of nutraceuticals. This study was therefore designed to evaluate the prescribing trend of nutraceuticals in government medical college and hospital in Aurangabad city of Maharashtra. This review gives highlights of some important facts regarding the use of nutraceuticals as the commercial and traditional remedies.

\section{METHODS}

This was an observational study conducted on patients of pediatrics outpatient department at government medical college and hospital Aurangabad over a period of 5 months from November 2018 to march 2019. The present study was taken up with the objectives of studying the prescription pattern of nutraceuticals among children under 12 years of age and to study whether the nutraceuticals are prescribed in generic or brand name by doctors in paediatric out patient department of government medical college and hospital Aurangabad, Maharashtra, India.

The study was conducted after obtaining clearance from institutional ethics committee in government medical college Aurangabad and is in accordance with the declaration of Helsinki.

Total 156 patients were enrolled in this study. The patients included in the study were patients of the Paediatric out patient department of government medical college and hospital Aurangabad, whose parents gave consent to participate in the study. Age group of patients included in this study was 1-12 years. This study was conducted after obtaining written, informed and understood consent from the parents of patients. Parents of patients also consented for the data to be published and reported. Pediatric patients more than 12 years old and patients who have not given consent were excluded from the study. The parents of patients were interviewed and the prescriptions were scrutinized to understand the usage of nutraceuticals. The data was entered into excel sheet and analysed based on percentages.

\section{RESULTS}

As depicted in (Figure 1), 75 male patients in paediatric department received of prescriptions contained nutraceuticals of one or the other types. However, 81 female patients in paediatric department received of prescriptions contained nutraceuticals.

As depicted in (Figure 2), 51 patients were below the age of 6 years who were prescribed nutraceuticals and 105 patients were above the age of 6 years who were given 
nutraceuticals. Patients below 6 years and who were not consuming tablets were given syrups.

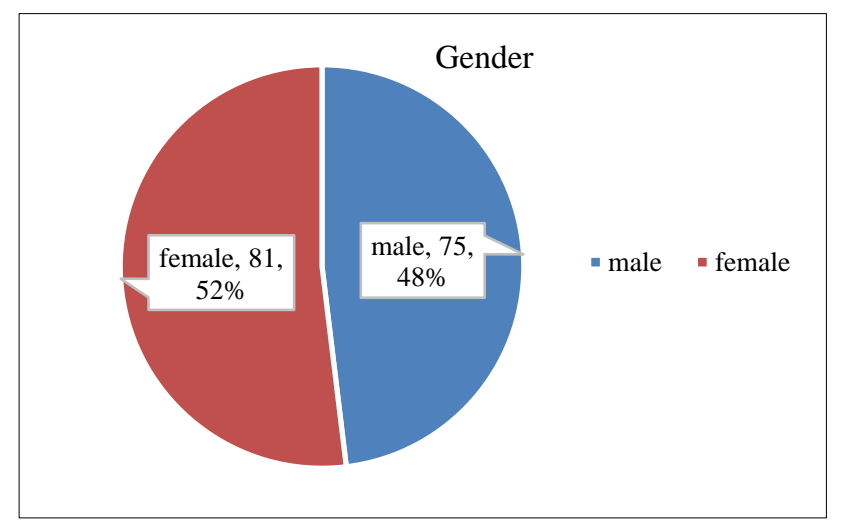

Figure 1: Distribution of male and female patients in study.

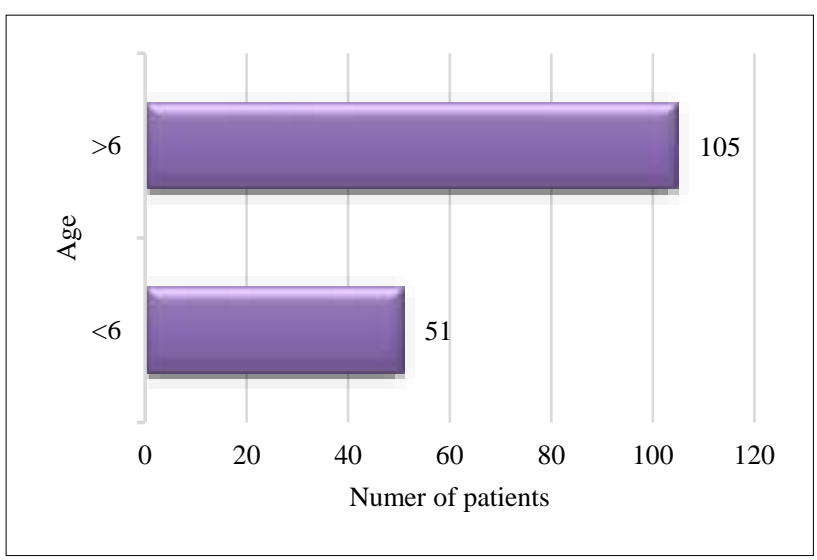

Figure 2: Distribution of under 6 and above 6 years patients in this study.

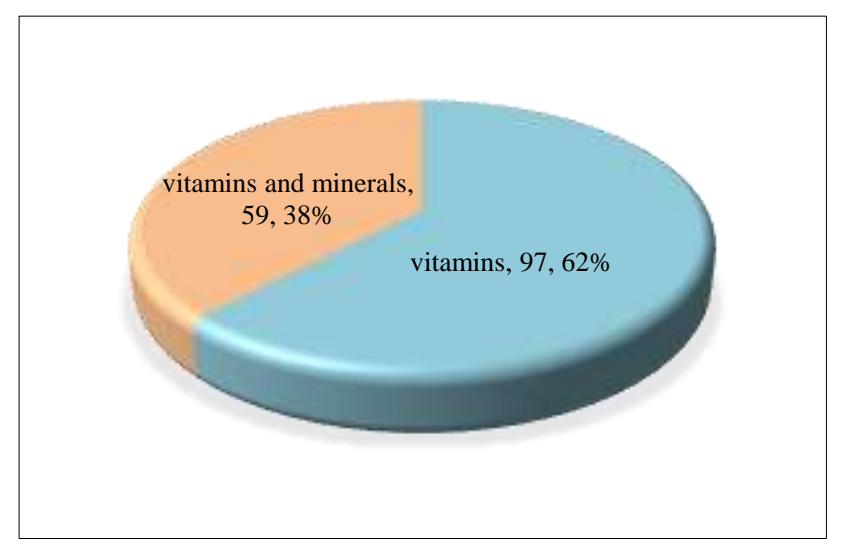

Figure 3: Distribution of vitamins, minerals, proteins and vitamin and minerals in paediatric patients.

Figure 3 shows the distribution of vitamins, minerals, proteins and combination of vitamin and minerals in paediatric patients. Vitamins include vitamin B complex, folic acid $5 \mathrm{mg}$ tablet and poly-vitamin tablet. Vitamins are given in 97 patients out of 156 patients. Proteins supplements are not given. Minerals are not given alone, they are given in combination with vitamins as combination of ferrous sulfate and folic acid tablet, calcium lactate with vitamin $\mathrm{D}$ tablet and calcium carbonate with vitamin D3 tablet. Vitamin and minerals are given in 59 patients out of 156 patients.

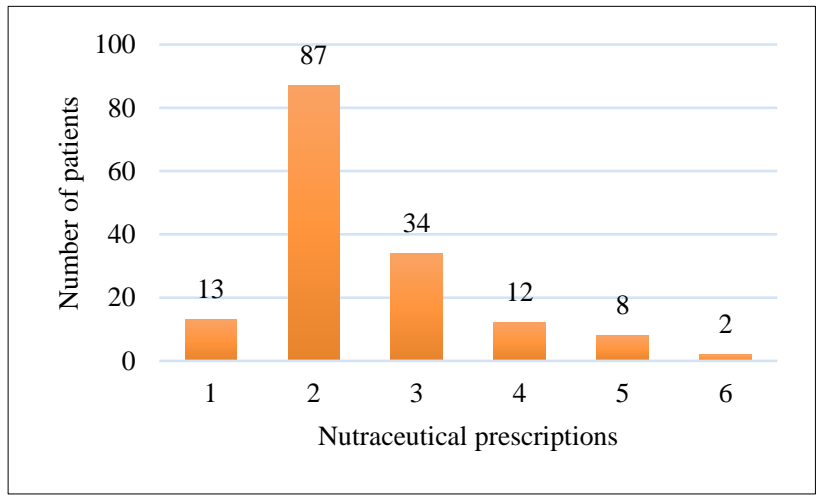

Figure 4: Distribution of various nutraceuticals in paediatric patients.

1- ferrous sulfate + folic acid tablet, 2- B complex, 3- calcium lactate + vitamin D tablet, 4- calcium carbonate + vitamin D3, 5folic acid tablet, 6- poly-vitamin tablet.

Figure 4 shows the distribution of various nutraceuticals in paediatric department. Ferrous sulfate + folic acid supplements were prescribed in 13 patients out of 156 patients. 87 out of 156 patients were prescribed B complex tablet. 34 patients were prescribed calcium lactate + vitamin D tablet out of 156 patients. 12 patients out of 156 were prescribed calcium carbonate + vitamin D3 tablets. 8 patients out of 156 were prescribed Folic acid Tablet and 2 patients out of 156 were prescribed poly-vitamin tablet.

In government medical college and hospital Aurangabad, all prescriptions were given in generic names. Brand name prescriptions were not given.

\section{DISCUSSION}

This study revealed that, from November 2018 to March 2019, total 156 patients were prescribed nutraceuticals in paediatric out patient department of government medical college Aurangabad, of which $51.92 \%$ female patients received nutraceuticals and $48.78 \%$ male patients received nutraceuticals. in study conducted by Osarenmwinda et al, total 1222 patients were evaluated in paediatric out patient department of which 649 were males that is $53.1 \%$ and females were 573 that is $46.9 \%{ }^{4}$

In this study, $62 \%$ patients received vitamins and $38 \%$ patients received vitamins and minerals. In a study conducted by Zaman TU et al, they concluded that $60 \%$ which is similar to this study patient were prescribed vitamins and $13 \%$ minerals were prescribed minerals. ${ }^{5}$ 
A total $67.31 \%$ patient receiving nutraceuticals were above the age of 6 years and $32.69 \%$ patients were under the age of 6 years. in study conducted by Osarenmwinda et al, 944 patients were under 6 years that is $77.4 \% .^{4}$

Among patients receiving nutraceuticals B complex was received maximum that is $55.77 \%$. After that calcium lactate with vitamin D tablet were prescribed $21.80 \%$. Combination of ferrous sulfate with folic acid was received by $8.33 \%$ patients. Calcium carbonate + vitamin D3 were received by $7.69 \%$ patients. Folic acid tablet was received by $5.13 \%$ patients. And least percentage of nutraceuticals were prescribed were poly-vitamin tablet that is $1.28 \%$.

All the drugs were prescribed in generic names that is $100 \%$ prescriptions were prescribed in generic names. Brand name prescriptions were $0 \%$. in study conducted by Osarenmwinda et al, drugs prescribed in generic name was $15.50 \% .^{4}$

\section{CONCLUSION}

Nutraceuticals are being increasingly perceived as beneficial to health and are being continuously used in the treatment. Majority of patients receiving treatment in the hospital expect to receive prescriptions for nutraceuticals. The future of nutraceuticals is bright and can transform healthcare in a developing country like India which utilizes a large number of natural compounds. More studies need to be taken up on prescription pattern of nutraceuticals in healthcare.

\section{ACKNOWLEDGEMENTS}

Authors would like to thank Dr. Prabha Khaire, Professor and head of department, department of paediatrics', Government Medical College, Aurangabad for assistance in the study.

Funding: No funding sources

Conflict of interest: None declared

Ethical approval: The study was approved by the Institutional Ethics Committee

\section{REFERENCES}

1. Gosavi S, Subramanian M, Reddy R, Shet BL. A Study of prescription pattern of neutraceuticals, knowledge of the patients and cost in a tertiary care hospital. J Clin Diag Res. 2016;10(4):2014-7.

2. Bhowmik D, Gopinath H, Kumar BP, Duraivel S, Kumar KPS. Nutraceutical - a bright scope and opportunity of Indian healthcare market. The Pharma J. 2013;1:29-41.

3. Rajasekaran A, Xavier R. Nutraceuticals as therapeutic agents: a review. Res J Pharm Tech. 2015;1:320-30.

4. Osarenmwinda IM, Erah PO. Prescribing trend of vitamins and other dietary supplements in tertiary health facility in Benin City. J Sci Pract Pharma. 2015;2:2-7.

5. Zaman TU, Adetunji H, Fadil E, Salih M, Salih M. Nutraceuticals: a slow transition from preventive to curative healthcare and the perceptions among physicians and patients-a study of south Delhi in India. Int J Pharma Sci Res. 2017;8(7):3-8.

6. Chiba T, Sato Y, Nakanishi T, Yokotani K, Suzuki S, Umegaki K. Inappropriate usage of dietary supplements in patients by miscommunication with physicians in Japan. Nutr. 2014;6:5392-4.

7. Kumari M, Jain S, Singh J. Research paper nutraceutical-medicine of future. J Global Bios. 2015;4(7):2790-4.

8. Radhika PR, Brij R, Singh M, Sivakumar T. nutraceuticals:an area of tremoundous scope. Int $\mathbf{J}$ Res Ayurveda Pharma. 2011;2(2):410-5.

9. Dureja H, Kaushik D. Development in nutraceuticals. Indian J Pharmacol. 2014;35:363-72.

10. Pandey M, Saraf SA. Nutraceuticals: new era of medicine and health. Asian J Pharma Clin Res. 2014;3:11-5.

11. Ahad T, Nissar J, Rather AH. Nutraceuticals-health benefits: a review. Int J Engineer Technol Sci Res. 2017;4(12):262-7.

12. Das L, Bhaumik E, Raychaudhuri U. Role of nutraceuticals in human health. J Food Sci Technol. 2012;49:173-83.

13. Radimer K, Bindewald B, Hughes J, Ervin B, Picciano MF. Dietary supplement use by US adults: data from the National Health and Nutrition Examination Survey, 1999-2000. Am J Epidemiol. 2004;160(4):339-49.

14. Patil CS. Current trends and future prospective of nutraceuticals in health promotion. Bioinfo Pharma Biotechnol. 2011;1(1):1-7.

Cite this article as: Kulkarni M, Ambekar P, Athawale S. Prescribing pattern of nutraceuticals in pediatric outpatient department at tertiary care teaching hospital. Int J Basic Clin Pharmacol 2020;9:1060-3. 